\title{
Pretreatment Lymph Node Metastasis as a Prognostic Significance in Cervical Cancer: Comparison between Disease Status
}

\author{
Soo Young Jeong, MD \\ Hyea Park, MD \\ Myeong Seon Kim, MD \\ Jun Hyeok Kang, MD \\ E Sun Paik, MD, PhD \\ Yoo-Young Lee, MD, PhD \\ Tae Joong Kim, MD, PhD \\ Jeong Won Lee, MD, PhD \\ Byoung-Gie Kim, MD, PhD \\ Duk Soo Bae, MD, PhD \\ Chel Hun Choi, MD, PhD
}

Department of Obstetrics and Gynecology, Samsung Medical Center, Sungkyunkwan University School of Medicine, Seoul, Korea

\author{
Correspondence: Chel Hun Choi, MD, PhD \\ Department of Obstetrics and Gynecology, \\ Samsung Medical Center, Sungkyunkwan \\ University School of Medicine, 81 Irwon-ro, \\ Gangnam-gu, Seoul 06351, Korea \\ Tel: 82-2-3410-3545 \\ Fax: 82-2-3410-0630 \\ E-mail: chelhun.choi@samsung.com \\ Received June 15, 2019 \\ Accepted October 28, 2019 \\ Published Online October 29, 2019 \\ *Soo Young Jeong and Hyea Park contributed \\ equally to this work.
}

\begin{abstract}
Purpose
Lymph node metastasis (LNM) is the most significant prognostic factor in cervical cancer that was recently incorporated into the International Federation of Gynecology and Obstetrics (FIGO) staging system. This study was performed to evaluate whether the prognostic significance of LNM differs according to disease status.
\end{abstract}

\section{Materials and Methods}

Patients with FIGO stage IB or higher cervical cancer who had pretreatment computed tomography and/or magnetic resonance imaging studies as well as long-term follow-up were enrolled in this retrospective study. The hazard ratio (HR) of Cox regression was used to determine the prognostic significance of LNM. The HRs were compared between the different tumor groups (based on stage, histology, tumor size, primary treatment, age, parametrium involvement, and lymphovascular space invasion).

\section{Results}

A total of 970 patients treated between January 1999 and December 2007 were included. The pretreatment LNM had prognostic significance in patients with stage IB1/IIA (HR for progression-free survival 2.10, $p=0.001$; HR for overall survival 1.99, $p=0.005$ ). However, the significance gradually decreased or disappeared with advancing stages. Similarly, the prognostic significance of the pretreatment LNM decreased with advancing disease status, including old age, parametrial involvement or lymphovascular space involvement. In contrast, the tumor size was associated with the prognostic significance of LNM with advancing status. The significance of the clinical LNM did not reflect the significance of the clinical stage. In contrast, the tumor size, parametrial involvement, and significance of the pathologic LNM reflected the clinical stage.

\section{Conclusion}

In patients with cervical cancer, pretreatment LNM on imaging has different clinical significance depending on the tumor status.

\section{Introduction}

Cervical cancer is common malignancy in female and the age-specific incidence rates of cervical cancer have a rising trend during 2019 in Korea [1]. In cervical cancer, the International Federation of Gynecology and Obstetrics (FIGO) had accepted the clinical staging system for a long time. This

\section{Key words}

Uterine cervical neoplasms, Neoplasm staging, Lymph nodes, Neoplasm metastasis, Diagnostic imaging, Prognosis staging system had strong prognostic significance. The clinical staging of cervical cancer is determined by examining the tumor size, vagina/parametrial involvement, or distant metastasis using the physical examination, colposcopy, cystoscopy, intravenous pyelogram, and radiography of the lung and skeleton. The FIGO Committee had adhered to the stance that clinical staging should be used in cervical cancer, because surgical staging is suitable only for early-stage dis- 
ease that may be possible to perform surgical treatment. Unfortunately, however, the majority of patients with cervical cancer are diagnosed with late stages, and are not surgical candidates [2].

Beside the clinical staging, other clinicopathological risks have additional prognostic meaning in cervical cancer. Lymph node metastasis (LNM) is the most significant factor in most studies [3]. In their extensive literature review, Creasman and Kohler [4] reported that surgically treated patients without any LNM had higher 5-year survival rates $(90 \%$ or higher) than did patients with pelvic LNM (50\% to 60\%). This group reported that lymph node involvement was an independent risk factor for survival [4]. Therefore, it is necessary to define an accurate cancer stage and to detect lymph node metastases for determining appropriate treatment and prognostication in patients with cervical cancer.

Because surgical staging is not a universal method, there is a practical limit to pathologically confirming LNM in all cervical cancer patients. Instead, clinical LNM can be used as surrogates for pathologic LNM. As imaging technology improves, computed tomography (CT) and magnetic resonance imaging (MRI) have enabled detection of lymph node metastases in patients with cervical cancer. With these methods, clinicians identify any enlargement of node size and morphologic information to determine node metastases. There are variable accuracy and CT had $>90 \%$ specificity and $<60 \%$ sensitivity, indicating that CT had high specificity in the detection of LNM. The sensitivity and specificity of MRI were $54 \%$ and $93 \%$, respectively [5].

With the alleged significance of LNM and improved imaging methodology for its detection, FIGO recently adopted LNM, including clinical LNM, as a staging system. However, LNM does not have the same prognostic significance in every disease state. It is critical to understand the relative importance of LNM in variable disease states of cervical cancer. Therefore, in this study, we assessed the relative importance of LNM in different disease states.

\section{Materials and Methods}

\section{Patients}

The inclusion criteria were as follows: (1) pathologically confirmed cervical cancer, (2) stage IB or higher according to the 2009 FIGO staging system, and (3) cancer evaluation using contrast-enhanced abdominopelvic CT or MRI prior to treatment initiation. A total of 970 patients were ultimately included.

Based on the 2009 FIGO staging criteria, all of the patients underwent bimanual pelvic and rectovaginal examination to identify the extent of disease. Routine laboratory testing and chest radiography were performed. Pretreatment imaging was also conducted to identify the status of the pretreatment LNM. Patients with stage IB to IIA cervical cancer were primarily treated with radical hysterectomy with or without adjuvant radiation or chemoradiation. Patients with stage IIB or higher cervical cancer were treated with concurrent chemoradiation or chemotherapy. After primary treatment with or without adjuvant treatment, all patients received adequate follow-up procedures. During this period, patients underwent physical examination, Pap smears, and tumor marker measurements every 3 months for the first 2 years, and every 6 months for the next 3 years. Imaging studies, such as chest radiography and abdominopelvic and/or chest $\mathrm{CT}$, were conducted every 6-12 months for the first 2 years and then annually for the next 3 years.

\section{CT or MRI image analysis}

All patients underwent contrast-enhanced abdominopelvic CT/MRI within 4 weeks before treatment initiation. Experienced radiologists interpreted these images. The clinical assessment was performed based on the radiological reports. According to their reports, 'Pretreatment $\mathrm{LNM}^{\prime}$ was defined as a short axis diameter of $\geq 1.0 \mathrm{~cm}$ on CT/MRI.

\section{Tumor groups for analysis}

Patients were divided into subgroups by several criteria. Patients were divided by age with a cutoff of 50 years. They were then divided into three groups by stage; "IB1/IIA," "IB2," and "IIB or higher." Tumor size was measured with clinical examination. If there were limited data, size measurement were made on imaging. The tumor size was divided into " $\leq 4 \mathrm{~cm}$ " and " $>4 \mathrm{~cm}$." Experienced pathologists performed histology on biopsy specimens that were obtained by biopsy or intraoperatively. We retrospectively assessed those pathologic reports. We analyzed the presence of cervical cancer cells, histologic types, parametrium involvement and lymphovascular space involvement (LVSI). The primary treatments were grouped as "operation alone," "operation with adjuvant/neoadjuvant radiation or chemotherapy," and "primary concurrent chemoradiation therapy."

\section{Statistical analysis}

The association between clinicopathologic parameters was examined using the chi-square test or Fisher exact test. Univariate Cox regression with hazard ratio (HR) was used to determine the prognostic significance of pretreatment LNM. The HR was compared between the different tumor groups 
Table 1. Clinicopathological characteristics of 970 patients

\begin{tabular}{|c|c|c|c|}
\hline & $\begin{array}{c}\text { Negative LNM on } \\
\text { imaging }(n=707)\end{array}$ & $\begin{array}{l}\text { Positive LNM on } \\
\text { imaging }(n=263)\end{array}$ & p-value \\
\hline \multicolumn{4}{|l|}{ Age (yr) } \\
\hline$<50$ & $343(48.5)$ & $119(45.2)$ & 0.405 \\
\hline$\geq 50$ & $364(51.5)$ & $144(54.8)$ & \\
\hline \multicolumn{4}{|l|}{ Stage } \\
\hline IB1 / IIA & $600(84.9)$ & $127(48.3)$ & $<0.001$ \\
\hline IB2 & $38(5.4)$ & $34(12.9)$ & \\
\hline IIB or higher & $69(9.8)$ & $102(38.8)$ & \\
\hline \multicolumn{4}{|l|}{ Histology } \\
\hline Squamous cell carcinoma & $547(77.4)$ & $225(85.6)$ & 0.007 \\
\hline Adenocarcinoma & $160(22.6)$ & $38(14.4)$ & \\
\hline \multicolumn{4}{|l|}{ Tumor size (cm) } \\
\hline$\leq 4$ & $588(86.5)$ & $102(40.3)$ & $<0.001$ \\
\hline$>4$ & $92(13.5)$ & $151(59.7)$ & \\
\hline \multicolumn{4}{|l|}{ Primary treatment } \\
\hline Operation alone & $351(49.6)$ & $32(12.2)$ & $<0.001$ \\
\hline Operation+adjuvant/Neoadjuvant treatment & $261(36.9)$ & $110(41.8)$ & \\
\hline Primary RT or CCRT & $95(13.4)$ & $121(46.0)$ & \\
\hline \multicolumn{4}{|l|}{ Year of treatment } \\
\hline$<2000$ & $200(28.3)$ & $37(14.1)$ & $<0.001$ \\
\hline$<2004$ & $240(33.9)$ & $104(39.5)$ & \\
\hline$<2008$ & $267(37.8)$ & $122(46.4)$ & \\
\hline \multicolumn{4}{|l|}{ Parametrial involvement ${ }^{a)}$} \\
\hline Negative & $572(93.5)$ & $118(83.1)$ & $<0.001$ \\
\hline Positive & $40(6.5)$ & $24(16.9)$ & \\
\hline \multicolumn{4}{|l|}{ Lymphovascular space invasion ${ }^{a}$} \\
\hline Negative & $302(68.2)$ & $166(76.1)$ & 0.042 \\
\hline Positive & $141(31.8)$ & $52(23.9)$ & \\
\hline
\end{tabular}

Values are presented as number (\%). LNM, lymph node metastasis; RT, radiation therapy; CCRT, concurrent chemoradiation therapy. a)Patients primarily treated with radical surgery.

(age, stage, histology, tumor size, primary treatment, parametrial involvement, and LVSI). A forest plot was used to display the HR (95\% confidence interval [CI]) of each patient group. The progression-free survival (PFS) and overall survival (OS) were estimated by using the Kaplan-Meier method. Each curve was compared using the log-rank test. The PFS was defined as the time interval from treatment to the first evidence of recurrence or to the last follow-up. The OS was defined as the time interval from diagnosis to the date of death or last follow-up. Statistical analysis was performed using R 3.3.2 (Vienna, Austria; http://www.R-project.org /). p-values of $<0.05$ were considered significant.

\section{Ethical statement}

This study was approved by the Institutional Review Board (IRB) of Samsung Medical Center (No. 2018-05-125).
The medical records of patients diagnosed with cervical cancer between January 1999 and December 2007 were retrospectively reviewed. Informed consent was waived.

\section{Results}

\section{Patient characteristics}

The clinicopathological characteristics of 970 patients included in this study are presented in Table 1. A total of 707 patients $(72.9 \%)$ had negative LNM on the pretreatment CT/MRI, while 263 patients $(27.1 \%)$ had positive LNM. As expected, the LNM positive group demonstrated more advanced stages and larger tumors than did the LNM nega- 


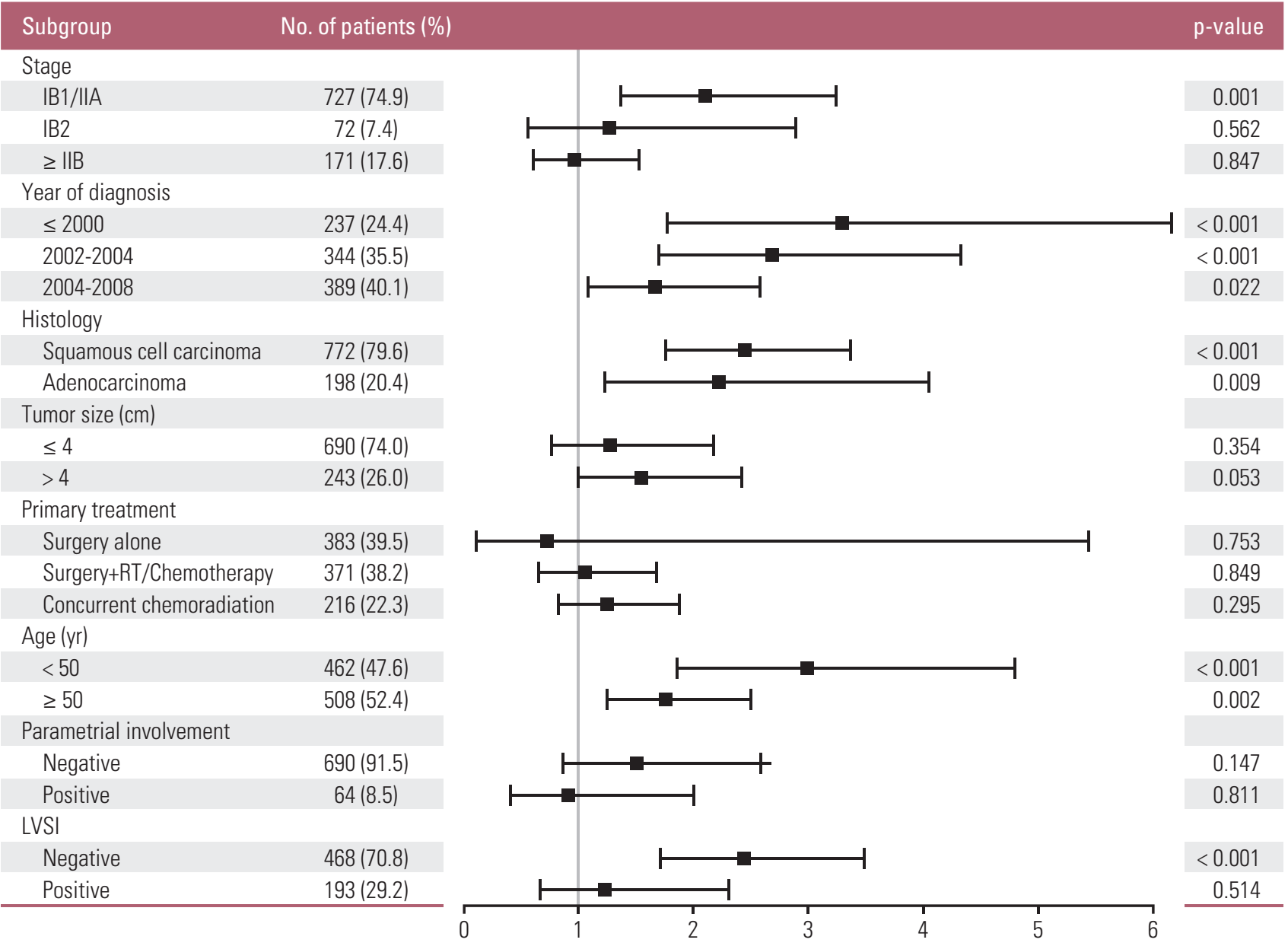

Fig. 1. Univariate Cox proportional hazard ratios for progression-free survival. RT, radiation therapy; LVSI, lymphovascular space invasion.

tive group ( $\mathrm{p}<0.001$ and $\mathrm{p}<0.001$, respectively). More patients received primary radiation therapy than primary surgery in the LNM positive group. Squamous cell carcinomas were more common in the LNM positive group than they were in the LNM negative group ( $85.6 \%$ vs. $77.4 \%$, $\mathrm{p}=0.007)$. Among the patients primarily treated with surgery, parametrial invasion was more common in the LNM positive group than it was in the LNM negative group (16.9\% vs. $6.5 \%$, p < 0.001).

\section{Prognostic significance of LNM by disease status}

The HR (95\% CI) of the LN metastasis for each patient subgroup is displayed in Fig. 1 and S1 Fig. Pretreatment LNM had prognostic significance in patients with stage IB1/ IIA (HR for PFS 2.10, $\mathrm{p}=0.001$; HR for OS 1.99, $\mathrm{p}=0.005$ ). This significance gradually declined or disappeared with advancing stage. Similarly, the prognostic significance of pretreatment
LNM decreased with more advanced disease status, which included factors such as old age, parametrial involvement, or LVSI. In contrast, tumor size showed trends of increasing prognostic significance for LNM with advancing disease status. LNM was only statistically significant in tumors that were $4 \mathrm{~cm}$ or larger.

To evaluate the effect of pretreatment LNM on prognosis, Kaplan-Meier curves in each subgroup were created. These curves included clinical stage, histology, tumor size, type of primary treatment, age, and parametrial involvement (Figs. 2 and 3). Although all patients with pretreatment LNM had worse prognosis than did those without pretreatment LNM, the magnitudes of the influence differed across the subgroups. The clinical stage, tumor size, type of primary treatment, and parametrial involvement were stronger prognostic factors than clinical LNM. The survival curves by clinical LNM did not cross the curves of each factor (Fig. 2 for PFS, S2A-S2D Fig. for OS). Interestingly, in patients who were pri- 
A

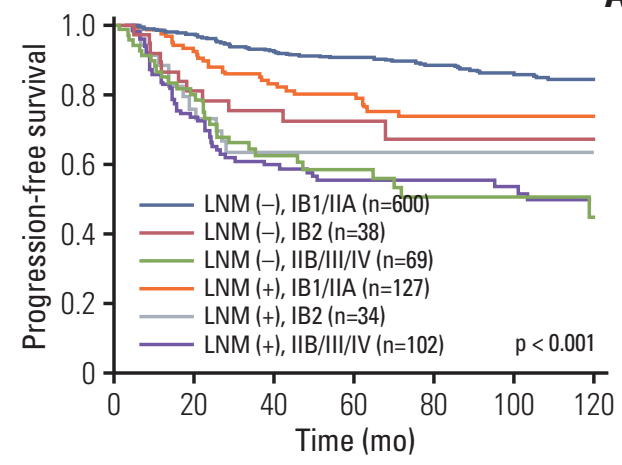

C

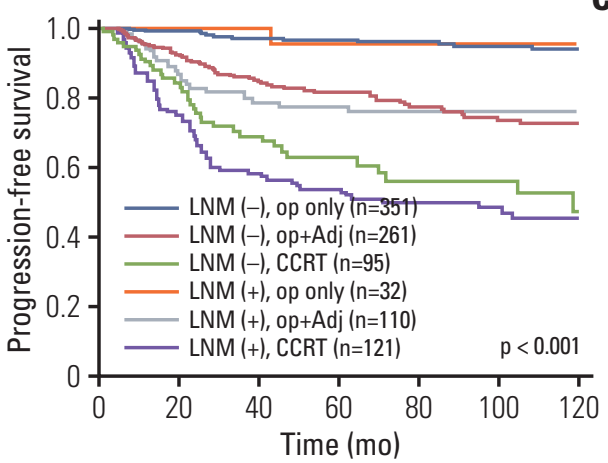

B

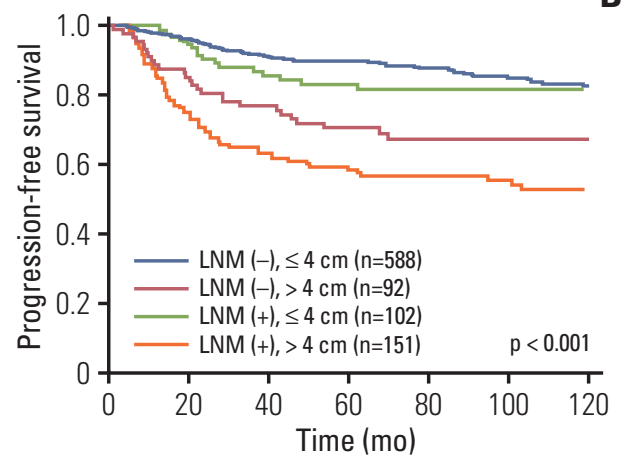

D

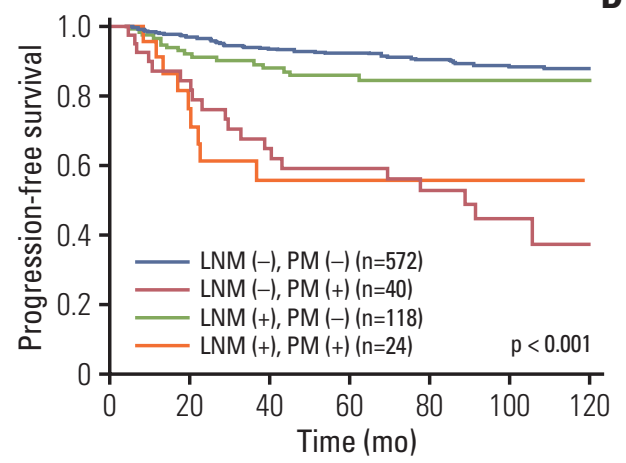

Fig. 2. Progression-free survival in patients with and without pretreatment lymph node metastasis (LNM) by subgroups. (A) International Federation of Gynecology and Obstetrics stage. (B) Tumor size. (C) Primary treatment. (D) Parametrium (PM) involvement. Op, operation; Adj, adjuvant therapy; CCRT, concurrent chemoradiation.

A

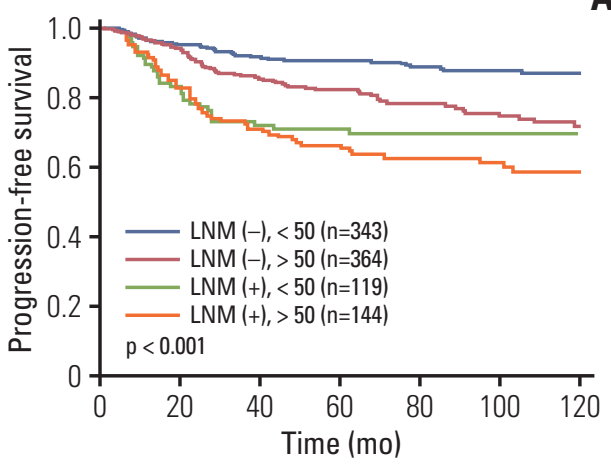

B

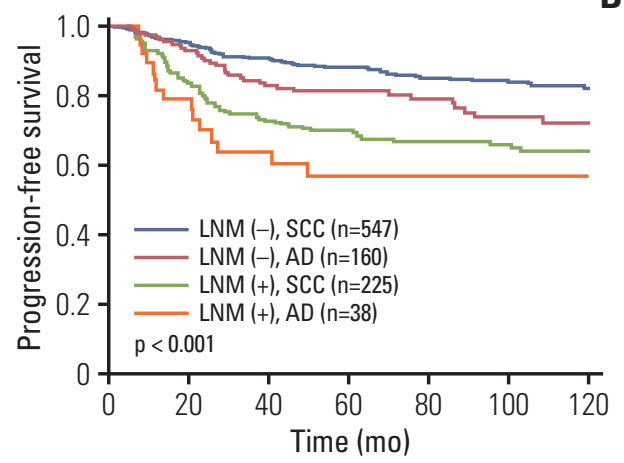

Fig. 3. Progression-free survival in patients with and without pretreatment lymph node metastasis (LNM) by subgroups. (A) Age. (B) Histology. SCC, squamous cell carcinoma; AD, adenocarcinoma.

marily treated with surgery, the survival curves by parametrial invasion were not influenced by the LNM status. Although histology and age have prognostic significance, this significance was weaker than that of the pretreatment LNM (Fig. 3 for PFS, S2E and S2F Fig. for OS).

\section{Risk of pathologic LNM by disease stage}

The imaging and pathologic results related to lymph nodes were not always consistent. Therefore, we also assessed the survival rates, PFS, and OS related to pathologic LNM. 

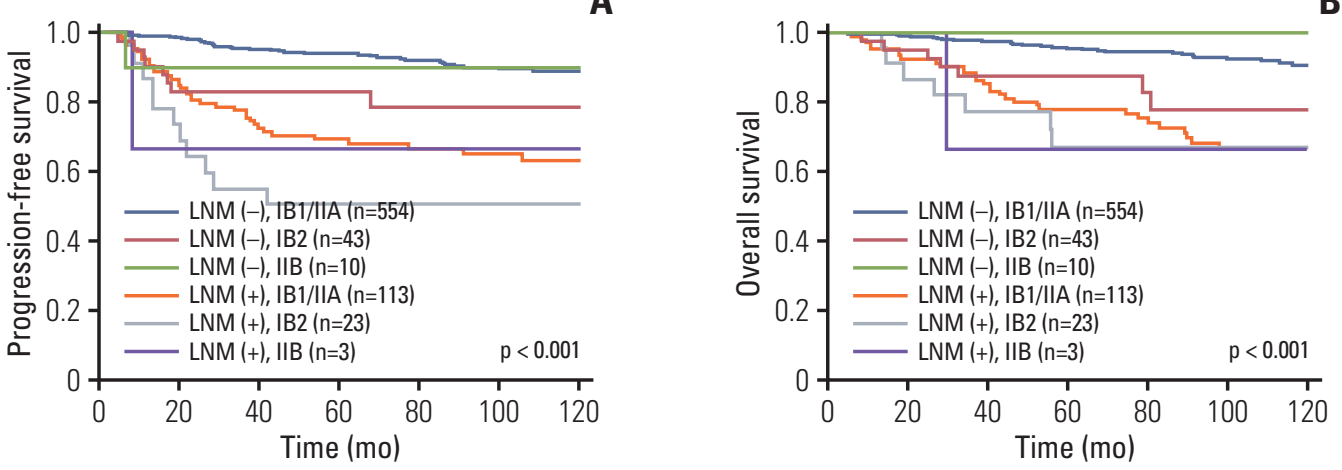

Fig. 4. Progression-free survival (A) and overall survival (B) in patients with and without pathologic lymph node metastasis (LNM) by cancer stage.

Table 2. Comparison pathologic and imaging results related to LNM

\begin{tabular}{lccc} 
Pathologic LNM & $\begin{array}{c}\text { Negative LNM on } \\
\text { imaging }(\mathbf{n}=606)\end{array}$ & $\begin{array}{c}\text { Positive LNM on } \\
\text { imaging }(\mathbf{n}=\mathbf{1 4 1})\end{array}$ & $\begin{array}{c}\text { p-value } \\
\text { Negative }\end{array}$ \\
Positive & $528(87.1)$ & $62(56.0)$ & $<0.001$ \\
\hline
\end{tabular}

Values are presented as number (\%). LNM, lymph node metastasis.

Fig. 4 demonstrates that positive pathologic LNM was more associated with survival rate than with clinical stage $(\mathrm{p}<$ $0.001)$.

Therefore, it is important to understand the expected concordance of imaging and pathologic results. The imaging assessment had a sensitivity, specificity, positive predictive value, and negative predictive value of $44.3 \%, 87.0 \%, 44.0 \%$, and $85.7 \%$, respectively (Table 2 ). This sensitivity was lower than that of other studies. From before 2000 to 2008, the sensitivity of CT/MRI imaging increased from $18 \%$ to $60 \%$. The clinical significance of pretreatment LNM has decreased with this increasing sensitivity (Fig. 1, year of diagnosis).

\section{Discussion}

We found that pretreatment LNM (by imaging) was a prognostic factor for survival in cervical cancer. Pretreatment LNM is influenced by multiple factors in different situations. For instance, the significance of pretreatment LNM was greater in patients with stage IB1/IIA disease than in those with higher stages. This relationship reflects the significance of imaging, especially in the early stages that may be ame- nable to surgery. In advanced disease, other factors are more likely to affect survival than the pretreatment LNM. While positive lymph nodes on imaging have prognostic value, they are less important than the stage and tumor size (Fig. 2A and B). The second point is that LNM (detected on pathology) influences the recurrence and mortality of cervical cancer more so than the clinical staging (Fig. 4). For example, patients with negative lymph nodes had better survival than those with positive lymph nodes, regardless of stage $(p<0.001)$. LNM on imaging did not influence the prognosis of cervical cancer as much as pathologic LNM. It is inevitable that pathologic LNM has a more powerful influence on survival than pretreatment LNM because current radiologic techniques cannot detect micrometastasis. In addition, pathologic results are more accurate and sophisticated than imaging findings.

The recent change to FIGO staging in cervical cancer reflects this importance of LNM. Prior to this revision, lymph node status was not included in the cervical cancer FIGO staging system. In developing countries, where the majority of cervical cancer patients live, surgery is typically not accessible due to economic difficulties [6]. Lymphangiography, one of invasive techniques, has also been used as a useful method for distinguishing LNM. However, lymphangiography is invasive and was found to have similar diagnostic 
rates as CT/MRI imaging in one meta-analysis [7]. Therefore, even though imaging results were not included in staging, many clinicians still used imaging techniques to identify the status of LNM. Many clinicians also suggested that the clinical examination does not always correlate with the pathologic results. Therefore, FIGO clinical staging results in a $20 \%-40 \%$ downstaging $[8,9]$. Some risk factors, such as adjuvant treatment, LVSI, or lymph node status, are not included in the cancer stage, even though they influence the prognosis within the same stage. Therefore, FIGO staging alone may not predict prognosis in patients with cervical cancer [10-13]. In two retrospective studies, risk assessment tools, including clinical histopathologic factors and LNM (detected by MRI or positron emission tomography $[\mathrm{PET}] / \mathrm{CT}$ ), were useful for predicting the prognosis of locally advanced cervical cancer (concordance index of new model vs. FIGO stage: 0.73 vs. 0.57 [13], 0.69 vs. 0.59 [12]). These modalities may be alternatives to FIGO clinical staging. In another study, a prognostic model combining radiologic findings, parametrial involvement defined by MRI, and LNM by PET/CT and clinical histopathologic factors provides more accurate prognostic information in all cervical cancer stages [11]. Eventually, the FIGO Gynecologic Oncology Committee revised the cervical cancer staging system in October 2018 [14]. The major change to this staging system is that patients are defined as "stage IIIC" if LNM is confirmed based on imaging or pathologic results.

Despite increasing importance of the LNM status, the best radiologic method to detect LNM remains unclear. As demonstrated in this study, both the diagnostic rate of pretreatment LNM and the sensitivity and specificity of CT/MRI have increased with time. These improvements are a result of the development of diagnostic tools such as helical CT, higher power Tesla MR machines, and diffusion-weighted imaging without contrast [15]. CT and MRI are the most widely used diagnostic tools for assessing metastatic lymph nodes. CT has a sensitivity of 30\%-80\% and a specificity of $>90 \%$ using the size criterion of $>1-1.5 \mathrm{~cm}$ to detect metastatic lymph nodes $[7,16]$. MRI has a sensitivity of $60 \%$ and a specificity of $>90 \%$ to detect metastatic lymph nodes [17]. Although MRI was superior to $\mathrm{CT}$ when evaluating the parametrium, tumor margin, and tumor size, there were no statistically significant differences between CT and MRI in defining the overall cancer status (including lymph node status) [9,18]. More sophisticated techniques have recently been introduced. In one prospective study, 3.0-T MRI had high diagnostic accuracy on the presurgical evaluation, although 3.0-T MRI was not significantly superior to the 1.5-T MRI [19]. In a meta-analysis, diffusion-weighted imaging (DWI) had a sensitivity of $83.3 \%-95.7 \%$ and a specificity of $74.7 \%-96.5 \%$. Therefore, DWI is useful in the detection of metastatic lymph nodes [20]. Many studies are currently underway to determine the accuracy of diagnostic tools or new techniques for detecting LNM.

This study has several limitations. Although a large number of patients was enrolled, this study was retrospective. Also, it seems unlikely that none of the scans performed between 1999 and 2007 had any technical issues or changes, and that the interpretation of several experienced radiologists were the same. In addition, stage IA cervical cancer patients were excluded from this analysis because we did not have enough of these patients to perform analyses.

CT and MRI are universal imaging techniques that are used to determine tumor extent and disease burden. Therefore, we chose to compare these two imaging modalities in this study. We found that the prognostic importance of pretreatment LNM on imaging is significant and has different clinical significance based on the tumor status. With new cervical cancer staging, pretreatment LNM can be used to individualize diagnosis and treatment. Nevertheless, it must be interpreted with caution.

\section{Electronic Supplementary Material}

Supplementary materials are available at Cancer Research and Treatment website (https:// www.e-crt.org).

\section{Conflicts of Interest}

Conflict of interest relevant to this article was not reported.

\section{References}

1. Jung KW, Won YJ, Kong HJ, Lee ES. Prediction of cancer incidence and mortality in Korea, 2019. Cancer Res Treat. 2019; 51:431-7.

2. Pecorelli S, Zigliani L, Odicino F. Revised FIGO staging for carcinoma of the cervix. Int J Gynaecol Obstet. 2009;105:107-8.

3. Lagasse LD, Creasman WT, Shingleton HM, Ford JH, Blessing
JA. Results and complications of operative staging in cervical cancer: experience of the Gynecologic Oncology Group. Gynecol Oncol. 1980;9:90-8.

4. Creasman WT, Kohler MF. Is lymph vascular space involvement an independent prognostic factor in early cervical cancer? Gynecol Oncol. 2004;92:525-9. 
5. Liu B, Gao S, Li S. A comprehensive comparison of CT, MRI, positron emission tomography or positron emission tomography/CT, and diffusion weighted imaging-MRI for detecting the lymph nodes metastases in patients with cervical cancer: a meta-analysis based on 67 studies. Gynecol Obstet Invest. 2017;82:209-22.

6. Torre LA, Bray F, Siegel RL, Ferlay J, Lortet-Tieulent J, Jemal A. Global cancer statistics, 2012. CA Cancer J Clin. 2015;65:87108.

7. Scheidler J, Hricak H, Yu KK, Subak L, Segal MR. Radiological evaluation of lymph node metastases in patients with cervical cancer: a meta-analysis. JAMA. 1997;278:1096-101.

8. LaPolla JP, Schlaerth JB, Gaddis O, Morrow CP. The influence of surgical staging on the evaluation and treatment of patients with cervical carcinoma. Gynecol Oncol. 1986;24:194-206.

9. Hricak H, Gatsonis C, Chi DS, Amendola MA, Brandt K, Schwartz LH, et al. Role of imaging in pretreatment evaluation of early invasive cervical cancer: results of the intergroup study American College of Radiology Imaging Network 6651Gynecologic Oncology Group 183. J Clin Oncol. 2005;23:932937.

10. Thomeer MG, Gerestein C, Spronk S, van Doorn HC, van der Ham E, Hunink MG. Clinical examination versus magnetic resonance imaging in the pretreatment staging of cervical carcinoma: systematic review and meta-analysis. Eur Radiol. 2013;23:2005-18.

11. Sala E, Micco M, Burger IA, Yakar D, Kollmeier MA, Goldman DA, et al. Complementary prognostic value of pelvic magnetic resonance imaging and whole-body fluorodeoxyglucose positron emission tomography/computed tomography in the pretreatment assessment of patients with cervical cancer. Int J Gynecol Cancer. 2015;25:1461-7.

12. Shim SH, Lee SW, Park JY, Kim YS, Kim DY, Kim JH, et al.
Risk assessment model for overall survival in patients with locally advanced cervical cancer treated with definitive concurrent chemoradiotherapy. Gynecol Oncol. 2013;128:54-9.

13. Kang S, Nam BH, Park JY, Seo SS, Ryu SY, Kim JW, et al. Risk assessment tool for distant recurrence after platinum-based concurrent chemoradiation in patients with locally advanced cervical cancer: a Korean gynecologic oncology group study. J Clin Oncol. 2012;30:2369-74.

14. Bhatla N, Aoki D, Sharma DN, Sankaranarayanan R. Cancer of the cervix uteri. Int J Gynaecol Obstet. 2018;143 Suppl 2:2236.

15. Lai CH, Yen TC, Ng KK. Surgical and radiologic staging of cervical cancer. Curr Opin Obstet Gynecol. 2010;22:15-20.

16. Wu SY, Huang EY, Chanchien CC, Lin H, Wang CJ, Sun LM, et al. Prognostic factors associated with radiotherapy for cervical cancer with computed tomography-detected para-aortic lymph node metastasis. J Radiat Res. 2014;55:129-38.

17. Sakuragi N. Up-to-date management of lymph node metastasis and the role of tailored lymphadenectomy in cervical cancer. Int J Clin Oncol. 2007;12:165-75.

18. Mitchell DG, Snyder B, Coakley F, Reinhold C, Thomas G, Amendola MA, et al. Early invasive cervical cancer: MRI and CT predictors of lymphatic metastases in the ACRIN 6651/ GOG 183 intergroup study. Gynecol Oncol. 2009;112:95-103.

19. Hori M, Kim T, Murakami T, Imaoka I, Onishi H, Tomoda K, et al. Uterine cervical carcinoma: preoperative staging with 3.0-T MR imaging: comparison with 1.5-T MR imaging. Radiology. 2009;251:96-104.

20. Shen G, Zhou H, Jia Z, Deng H. Diagnostic performance of diffusion-weighted MRI for detection of pelvic metastatic lymph nodes in patients with cervical cancer: a systematic review and meta-analysis. Br J Radiol. 2015;88:20150063. 